\title{
Estrategias estatales para las Inversiones Extranjeras Directas ¿Qué puede aprender Argentina de las reformas económicas en China?
}

\author{
Gonzalo Ghiggino**
}

\section{Resumen}

Tras el fin de la Guerra Fría y ante el impulso expansivo del capitalismo global, los países en vías de desarrollo iniciarán un proceso de reformas tanto estatales como económicas. Estas reformas se enmarcan en el proceso de globalización económica que tuvo su apogeo con la incorporación de nuevos mercados luego de 1991. Uno de los factores de este impulso a la globalización serán las Inversiones Extranjeras Directas (IED). Existe pues una relación directa de mutua correspondencia entre la globalización económica y las IED. Las IED tendrán una rápida expansión gracias a la globalización al tiempo que estas ayudaron al proceso globalizador. En este orden la discusión sobre el rol del Estado ante el impulso de la globalización será decisiva. La presencia o no de un Estado considerado "neoliberal" serán las variantes que se analizan en el presente texto dado que el rol del Estado en la promoción del desarrollo y el fomento de las IED será clave para el éxito o fracaso de las políticas económicas en China y Argentina.

Palabras claves: China, Argentina, IED, reforma del Estado, reformas económicas.

\begin{abstract}
After the end of the Cold War, the developing countries will begin a process of economic and state reforms facing the expansionary impulse of capitalism. This reform is part of the process of economic globalization that had its apogee with the incorporation of new markets after 1991. One of the factors of this impulse to economic globalization will be Foreign Direct Investments (FDI). There is therefore a direct relationship of mutual correspondence between economic globalization and FDI. The FDI had a rapid expansion thanks to globalization, while they helped the globalization process. In this order, the discussion on the role of the State in the face of economic globalization will be decisive. The presence or not of a State considered "neoliberal" will be the variants that are analyzed in this text, considering that the role of the State in the promotion of development and promotion of FDI will be the key in the success or failure of economic policies in China and Argentina.
\end{abstract}

Keywords: China, Argentina, FDI, state reform, economic reform.

\footnotetext{
* Research Fellow at Shanghai University (China) \& CIMI-CONICET (Argentina). Email gonzaloghiggino606@hotmail.com. Received March 7th 2019; accepted: April 23 2019.
} 
Gonzalo Ghiggino

Estrategias estatales para las Inversiones Extranjeras Directas ¿Qué puede aprender Argentina de las reformas económicas en China?

\section{Introducción}

El concepto de neoliberalismo entendido como una particular ideología económico-política, difundida en Europa y Estados Unidos entre la década del ochenta y noventa, propuso que los Estados debían cumplir un rol menor en la economía (Harvey, 2007). Este modelo de Estado débil fue aplicado en América del Sur, en contextos dictatoriales (Chile en 1973, Argentina en 1976) y luego por gobiernos democráticos en la década de los noventa. Durante el mismo período China emprendió medidas reformistas manteniendo, por el contrario, un Estado fuerte. El proceso iniciado en 1978 y profundizado luego de 1992 no significó el alejamiento o achicamiento del Estado sino más bien su reconfiguración y modernización acorde a los intereses del plan de reformas que el gobierno promovía.

Se entiende en el presente trabajo que el Estado juega un rol determinante en el proceso de desarrollo económico, y las inversiones extranjeras (IED) pueden ser claves. Para ello las reformas post 1991 llevadas cabo por los países emergentes han sido la herramienta para atraer inversiones necesarias para el desarrollo. Estas reformas se darán en el marco del fin de la Guerra Fría que implicó la propagación de las teorías neoliberales como doctrina para las reformas estatales.

Tenemos entonces, en este contexto, una serie de reformas estatales en los países emergentes inducidas por teorías neoliberales. Desde China hasta la Argentina estas políticas fueron entendidas como parte de un mismo proceso (Venugopal, 2015). Por lo tanto, acorde a lo que se pude interpretar, tanto China como Argentina, implementaron políticas neoliberales de reformas del Estado pero que finalmente darán distintos resultados. Para el caso de China logró un estadio de desarrollo económico con sus reformas y políticas para las IED en tanto que Argentina fracasó rotundamente. ¿Se puede decir entonces que se trata de dos caras de la misma moneda?

Lo que se plantea aquí es que ambos procesos de reforma estatal pueden ser comparables en el marco en que se dieron durante los años noventa, tras el fin de la Guerra Fría y el advenimiento del capitalismo y la doctrina neoliberal como dogmas incuestionables ${ }^{1}$. Pero como veremos finalmente, estos procesos distan de ser similares con resultados diametralmente opuestos.

Por lo tanto, a partir del análisis histórico, este artículo se centra en las medidas emprendidas por los Estados chino y argentino para la atracción de IED en el marco de post Guerra Fría. Las reformas económicas en Argentina fueron (durante los años noventa) "ejemplo" de los que los países emergentes debían hacer, en tanto que las reformas en China se daban en un contexto de profundización de la apertura económica que el gobierno de Deng Xiaoping había iniciado en 1979.

Estas reformas paradigmáticas, serán claves para el fracaso y el éxito en uno y otro caso. Se observa que, a partir de 1992, las medidas de incentivo y planificación lejos de disminuir el rol del Estado en China afianzaron su papel como actor económico clave. En tanto que en Argentina la reducción y debilitamiento del Estado, así como la ausencia de políticas y estrategias para las IED, producto de las reformas iniciadas a comienzos de los noventa, serán en parte responsables de la crisis de 2001.

${ }^{1}$ En este sentido es interesante citar la famosa obra del académico norteamericano Francis Fukuyama y su obra de 1992 "El fin de la Historia y el Ultimo Hombre", donde pregona el fin de la ideología y el advenimiento del capitalismo como doctrina económica incuestionable a nivel global. Ver Fukuyama (1992). 
Indudablemente los países emergentes requerían, y en el caso de Argentina aun requieren, de reformas para el mejor funcionamiento del Estado y que éste sea capaz de diseñar políticas de largo plazo para alcanzar el desarrollo económico. Si bien el trasfondo es distinto, ambos casos, tuvieron puntos de partidas similares, el caso chino demostró que el Estado siempre debe estar presente en el diseño de políticas estatales sobre todo en la dirección de las IED. Mientras que el caso argentino demostró, precisamente que la ausencia del Estado y la falta de políticas puede llevar al fracaso de todo intento de desarrollo.

Luego de más de 20 años de reformas, China ha logrado un desarrollo económico consolidado, y las IED jugaron un papel clave gracias al funcionamiento del Estado, por su parte Argentina sigue su camino pendular con períodos de crisis recurrentes, sin poder consolidar un proceso de desarrollo sostenido. Por lo tanto, entender el rol del Estado y cuán importante son las políticas estatales es fundamental para romper el círculo vicioso de crisis en las que el país se ha visto envuelto en su historia reciente.

Basándonos en un análisis histórico-comparativo de los primeros años de reforma estatal, se analizarán en una primera parte, las reformas del Estado en Argentina y en China en un contexto de fin de Guerra Fría y cambio en el orden internacional. Para luego realizar un análisis de las estrategias estatales en ambos países a partir de dichas reformas, y finalmente concluir con los resultados de ambas reformas y las estrategias aplicadas, y por qué el caso chino es paradigmático por su éxito.

\section{Las reformas del Estado: América Latina y el caso argentino}

La crisis de los años ochenta llevó a los países latinoamericanos a replantear el rol del Estado en la economía. El modelo de Industrialización por Sustitución de Importaciones (ISI), vigente desde los años cincuenta, ya no tendría más lugar en las políticas estatales. El final de las políticas de sustitución de importaciones junto con el surgimiento de un nuevo orden internacional tras el fin de la Guerra Fría presagiaron un cambio de época signado por el advenimiento de la democracia liberal y el libre mercado como dogmas incuestionables (Fukuyama, 1992).

Se admitió entonces que el Estado interventor era el problema y se planteó su reducción como solución a la crisis. Así entre 1990 y 1995 se llevaron a cabo en Latinoamérica una serie de reformas estructurales tendientes a reducir el "peso del Estado en la economía" (Cruz Soto, 2002). Los gobiernos surgidos tras las elecciones de fines de los años ochenta en el marco de la crisis fueron quienes llevaron a cabo estas políticas de reforma, acorde a lo que en el contexto internacional se promulgaba (Brooks \& Wohlforth, 2001).

La idea de "modernizar el Estado" ganó fuerza en América Latina y gobiernos como los de Carlos Menem en Argentina, Fernando Collor de Melo en Brasil, Carlos Salinas de Gortari en México, Alberto Fujimori en Perú y Carlos Andrés Pérez en Venezuela fueron paradigmáticos por sus políticas neoliberales. Así, el Estado en América Latina delegó su función de promotor del desarrollo.

La necesidad de obtener préstamos internacionales y la obligación al pago de la deuda externa llevó a los gobiernos latinoamericanos a la reestructuración de sus deudas externas mediante el Plan Brady y con 
Estrategias estatales para las Inversiones Extranjeras Directas ¿Qué puede aprender Argentina de las reformas económicas en China?

ello la consecuente aceptación de los postulados del Consenso de Washington que guiarían el proceso de reforma del estado y la economía a partir de 1990 (Benecke \& Nascimento, 2003).

Los postulados del Consenso de Washington se basaron en diez puntos: 1) disciplina fiscal, 2) redirección del gasto público, 3) reforma tributaria 4) tasas de interés determinadas por el mercado, 5) tipo de cambio competitivo, 6) liberalización del comercio, 7) liberalización de barreras para las inversiones extranjeras, 8) privatizaciones de empresas públicas, 9) desregulación financiera y 10) seguridad jurídica (Benecke \& Nascimento, 2003). Bajo estas condiciones los gobiernos latinoamericanos comenzaron sus reformas que, aunque similares en su contexto, tuvieron una aplicación que varió acorde a la realidad de cada país. Por ende, si bien el resultado de estas políticas está lejos de ser positivo, tuvo efectos dispares.

En Argentina la reforma del Estado comenzó con la crisis hiperinflacionaria de 1989. Tras una hiperinflación incontrolable y una crisis económica sin precedentes hasta el momento, el gobierno de Carlos Menem impulsó la reforma del Estado como marco para las demás reformas que se llevarán a cabo años después. En agosto de 1989 se aprueba la ley 23.696 de Reforma del Estado. Esta ley autorizaba al gobierno a proceder con la privatización de empresas estatales y a la fusión y disolución de entes públicos ${ }^{2}$.

Esta ley será la matriz de todas las políticas neoliberales implementadas a partir de 1989 en la Argentina 3 . La posibilidad de reformar el estado brindó al gobierno de Menem las herramientas claves para llevar cabo las reformas en profundidad en sectores del estado y la economía que hasta entonces se habían mantenido intocables. Se trató de una herramienta que permitió realizar el cambio estructural que modificará profundamente el funcionamiento y alcance del estado.

Uno de los objetivos por otra parte fue la de disciplinar al empresariado nacional. Tras años de negocios con el Estado, cierto sector nacional denominado "patria contratista", había cooptado los negocios con el Estado haciendo de las obras públicas y los sobreprecios pagados por el Estado su principal fuente de ingreso (Escudé, 2006). Consecuentemente, gran parte de este empresariado se oponía tanto a las privatizaciones como a la entrada de competencia ante una eventual apertura económica. Fue, curiosamente, el sector privado local el que se opuso a cualquier intento de reforma del Estado por parte del gobierno.

Así las políticas de reforma y apertura por parte del gobierno argentino se orientaron tanto hacia el frente interno como el frente externo. Con las reformas, el gobierno buscaba por un lado neutralizar los negocios del empresariado con el Estado nacional y por el otro "modernizar al Estado" y abrir la economía para avanzar a una economía plena de mercado facilitando la llegada de inversiones extranjeras y nuevos competidores al mercado local, al tiempo que se pretendía cumplir con los pagos de deuda (Basualdo, 2006).

Por ello las reformas del Estado estuvieron orientadas prácticamente a reducir a su mínima expresión al Estado y sus funciones, y garantizar seguridad y rentabilidad a las empresas extranjeras que llevaran

\footnotetext{
${ }^{2}$ Ley 23.696 de Reforma del Estado, Ministerio de Economía de la Nación

${ }^{3}$ Si bien la ley de reforma del Estado data de 1989, las reformas tardaron en aplicarse siendo el punto de partida el año 1991 cuando se aprueba la ley de convertibilidad que fijaba el valor del peso al dólar, y se aprueban una serie de privatizaciones de empresas estatales.
} 
inversiones a la Argentina. De esta manera el Estado se convirtió en garante para las inversiones extranjeras al tiempo que renunciaba a ser el Estado desarrollista que se venía promoviendo desde los años cincuenta.

\section{Las reformas post 1992 en China}

Luego de más de diez años de reformas y aperturas graduales, a comienzos de la década de 1990 se realizarán cambios estructurales en las burocracias chinas, principalmente relacionadas con las agencias de planificación. El tercer período de reformas iniciado por Deng Xiaoping hacia 1992 durante el viaje por el sur, se focalizó en las leyes y regulaciones para acelerar los cambios en las empresas de propiedad del Estado (State Own Enterprises) ${ }^{4}$.

Las leyes emitidas por Deng tras su viaje por el sur fueron: "Regulación sobre la transformación del mecanismo operativo de las empresas propiedad de todo el pueblo", "Regulaciones sobre la supervisión y el control de los activos estatales", "Ley de corporaciones", y "Decisión del comité central del PCCh sobre algunos asuntos relacionados con el establecimiento de una estructura económica de mercado socialista" (Wang, 1998).

Una de las características de este conjunto de reformas fue que el gobierno cambió sus reajustes de reformas parciales a una reforma completa. Todas esas leyes fueron diseñadas para establecer un sistema empresarial moderno, que coincidía con el requisito de la estructura económica de mercado socialista. Otra característica sobresaliente de este período fue que el gobierno desarrolló un nuevo concepto de reforma de la propiedad, que se pretendía fuera el núcleo de la reestructuración y el desarrollo económico. China adoptó una modelo de reformas económicas que se desarrollaba en dos sentidos: el sistema de planificación preexistente continuaba aplicándose mientras se realizaban experimentos económicos regionales, a gran escala, que resultaron satisfactorios (Huyghebaert \& Quan, 2011).

La globalización económica proporcionó un impulso y un fundamento importante a las reformas llevadas a cabo. Podría entenderse que la serie de reestructuraciones de la burocracia económica central china desde 1990 marca la retirada del Estado intervencionista considerándose como parte de un inevitable proceso de liberalización y desregulación económica. Sin embargo, incluso cuando se presentaron fuertes presiones externas para la reforma, la clase política nacional las utilizó para justificar su propia agenda. La opción de desmantelar el poderoso aparato económico estatal impulsado por parte del poder político, tal como se llevó a cabo en muchos países de occidente, no estaba destinada a la retirada del Estado. En el caso chino no se desmanteló ni llevó a cabo una desregulación en los hechos.

Las reformas no se llevaron a cabo para reducir una intervención burocrática en la economía, sino para redistribuir el poder y las funciones dentro de la burocracia económica. El Estado central chino persiguió un modo de intervención enfocado y eficiente, especialmente en áreas prioritarias. La discrepancia entre la fachada (retiro del Estado y desregulación) y el rol efectivo de las burocracias estatales sugieren que, a pesar del poder penetrante de la globalización económica y las presiones exógenas para la reforma económica, el Estado mantuvo su papel clave en la intervención de la economía (Jung, 2008).

\footnotetext{
${ }^{4}$ El primer período de reformas se llevó a cabo entre 1979 y 1984 considerado como experimental, se lo llamó "Las reformas iniciales". En tanto que el segundo período inciado en 1984 hasta 1992 serán "Las reformas graduales industriales".
} 
Gonzalo Ghiggino

Estrategias estatales para las Inversiones Extranjeras Directas ¿Qué puede aprender Argentina de las reformas económicas en China?

\section{Políticas estatales para la IED en Argentina}

Durante los años noventa la República Argentina vivió un auge de inversiones extranjeras. La Reforma del Estado iniciada en 1989 hizo posible el proceso de privatizaciones y las facilidades otorgadas a la IED. No hubo por parte del Estado una estrategia clara para orientar las IED, sino que se limitó a facilitar el proceso. Es decir, el Estado garantizó con nuevas leyes que las inversiones tendrían consideración especial acordando hacerse cargo, mediante convenios de arbitraje internacional, ante cualquier eventualidad que perjudicara a dichas inversiones (Granato \& Oddone, 2007).

El mensaje hacia afuera del país era claro y las compañías inversoras contarían con todas las facilidades y el apoyo del gobierno nacional. Si bien el país encaraba un proceso de apertura e inserción internacional manifestada en el alineamiento internacional con los Estados Unidos y de coordinación de políticas con los organismos internacionales como el FMI, las inversiones no encontraron atractivo el país como para aprovechar el comercio internacional.

Las IED se establecieron en el país dado las condiciones que a partir de los años noventa comenzaba a visibilizarse. Estas condiciones que marcaron una ruptura con el pasado fueron tres; primero, el marco regulatorio establecido a partir de las reformas del Estado, pues las empresas se sentían ahora con la seguridad de que no cambiaran las reglas de juego, segundo, el mercado interno, dado que ante las demandas y las perspectivas de crecimiento en el contexto de estabilización monetaria garantizarían un repunte del consumo y que ante el nuevo marco serían más favorables para las empresas internacionales que las locales, y tercero, el surgimiento del MERCOSUR, el proceso de integración y las bajas arancelarias auguraban un incremento de las exportaciones a los países del bloque en especial a Brasil con un mercado interno de más de 150 millones de habitantes (Porta \& Ramos, 2002).

Sin dudas ante esta transformación ocurrida las empresas trasnacionales comenzaron a jugar un papel cada vez más importante en la economía argentina. En este contexto aprovecharon con ventajas, frente a la mayoría de las empresas nacionales, las nuevas condiciones de competencia. Con estas condiciones tres oleadas sucesivas de IED se dirigieron al sector de los servicios públicos privatizados, la industria manufacturera y los servicios privados. Así la IED sumó más de 100.000 millones de dólares entre los años 1990 y 2000 (Porta \& Ramos, 2002). Al depender altamente de los ingresos de fondos extranjeros mediante inversiones la economía argentina inició un proceso de transformación que significó la transnacionalización y la dependencia cada vez mayor hacia el capital extranjero.

Dada la insuficiencia de capitales locales se necesitó de la entrada de capitales extranjeros para estimular la economía. Por otra parte, y más importante aún, el proceso privatizador funcionó como un imán para atraer la IED. Tal es así que entre 1990 y 1993 en el auge privatizador el 51 por ciento de las inversiones extranjeras directas corresponden a las privatizaciones de los activos públicos. Tras la venta parcial de la petrolera estatal Yacimientos Petrolíferos Fiscales (YPF) en 1993, el proceso de privatizaciones dejó de ser fuente central de ingresos de IED para dar ese lugar a las Fusiones y Adquisiciones (FyA) de empresas privadas, y en un nivel menor el financiamiento para las inversiones productivas ya sea para nuevos proyectos (greenfield) o para la ampliación de otros (Kulfas, Portas, \& Ramos, 2002). 
Analizando sectorialmente, las IED se destinaron en primer lugar al sector petrolero representando un 56 por ciento del total; en segundo lugar la industria manufacturera con el 23 por ciento del total, dentro de la industria manufacturera las inversiones se destinan principalmente a alimentos, bebidas y tabaco (31 por ciento), química caucho y plástico ( 29 por ciento) y automotriz y transporte (18 por ciento); finalmente en tercer lugar las IED se destinaron a los servicios públicos representando un 21 por ciento del total (Kulfas, Portas, et al., 2002).

La falta de una planificación estratégica para con las inversiones no solo se evidencia ante las facilidades otorgadas, sino que se hace presente ante la necesidad de asegurar el pago de la deuda tal como los organismos internacionales y los países acreedores habían planteado. La inclusión de bancos extranjeros en los consorcios adjudicatarios de las empresas privatizadas fue obligatoria. Los consorcios fueron conformados por tres partes; las empresas extranjeros, el socio local y el banco internacional que tenía en su poder bonos de deuda Argentina y que a su vez financiaba la compra (Kulfas, Porta, \& Ramos, 2002).

Las empresas locales en su mayoría participaron del proceso privatizador funcionando como lobistas entre las empresas internacionales y el gobierno nacional. Pero esta participación fue prácticamente efímera ya que, una vez consumada la privatización mediante la compra de la compañía estatal, las empresas locales se retiraron del negocio vendiendo su parte minoritaria dejando a la empresa extranjera con la totalidad del capital.

El sostenido ingreso de capitales generó no solo una alta dependencia de la economía argentina y su transnacionalización, sino que también la hizo más vulnerable a los cambios adversos de le economía internacional como sucederá para finales de los noventa. Por otra parte, la falta de políticas públicas orientadas a la recepción y redirección de la IED generó una fuerte desarticulación del aparato productivo nacional. Hacia fines de los años novenas resultarán evidentes las fallas del sistema económico, siendo las reformas económicas implementadas a partir de 1991, en parte responsables de estos problemas. El fracaso económico y la transnacionalización del sector productivo, así como la especulación financiera y la falta de respuestas del Estado argentino producto de su debilitamiento sistemático, llevaran a la debacle de los años 1998-2002 siendo el año 2001 el más recordado por su estallido económico y social. ${ }^{5}$

Los gobiernos surgidos en los años de post crisis implementarán una política diametralmente opuesta a la de los años noventa, y con una fuerte crítica al neoliberalismo y a las reformas estatales. Si bien se buscó, a partir de 2003, consolidar el rol del Estado, la política de intervención económica solo afectó el desempeño de las IED en el país, al tiempo que las respuestas a los vaivenes económicos fue una mayor intervención aún. Aunque esta intervención estatal fue entendida como la solución a los problemas de un Estado debilitado y a una economía trasnacionalizada, no significó como se pretendió, una consolidación real del aparato estatal, sino que solo fueron políticas anticíclicas que poco sirvieron para un real fortalecimiento del Estado.

\footnotetext{
5 A partir de la segunda mitad de los años 90s la economía Argentina tuvo impactos externos como la crisis del tequila (1995), crisis asiática (1997), crisis rusa (1998) y crisis brasilera (1999). La vulnerabilidad externa por la alta dependencia a los capitales externos producto de la falta de inversiones locales y la trasnacionalizacion económica, llevó al agravamiento de la crisis que de por sí misma se venía dando desde 1997. En 1998 el país entrará en una fuerte recesión que durará hasta el 2002. El estallido del año 2001 significó un punto de inflexión en la Argentina respecto a la importancia de integrarse al mundo siguiendo parámetros promovidos desde los centros financieros, como lo fueron las reformas estatales y la apertura económica.
} 
Gonzalo Ghiggino

Estrategias estatales para las Inversiones Extranjeras Directas ¿Qué puede aprender Argentina de las reformas económicas en China?

\section{Las estrategias estatales en China frente a las IED}

Por su parte las estrategias utilizadas por el gobierno chino estuvieron orientadas a captar aquellas inversiones destinadas al sector productivo y aquellas orientadas a las inversiones desde cero (greenfield), como a la asociación con las empresas locales mediante joint ventures (JV - asociaciones entre empresas locales y extranjeras). Las IED en China pasaron de unos 19 miles de millones de dólares en 1990 a unos 900 miles de millones en 1999 siendo el segundo receptor en el mundo y el primero entre los países emergentes (Kulfas, Porta, et al., 2002).

Hacia 1992, en el inicio de la tercera etapa de reformas iniciadas por el gobierno, las IED se focalizaron en bienes no transables. Dos años después, cerca del 64 por ciento de las IED se destinaron a los sectores exportables de las industrias (Long, 2005). La evolución de las IED se dio, principalmente, mediante JV y en menor medida asociaciones de cooperación y empresas sin ningún tipo de sociedad. Sin embargo, las compañías extranjeras sin ningún tipo de sociedad no tuvieron las mismas posibilidades que las JV, pues no pudieron, durante toda la década, adoptar tecnología avanzada ni equipamiento de avanzada o destinar la mayoría de su producción a la exportación, una política acorde a lo diseñado por el gobierno con el fin de desarrollar la competitividad de las empresas locales (Long, 2005).

Algunos de los factores por los que las empresas extranjeras aceptaron las condiciones para asentarse e invertir en China fueron el tamaño del mercado, el bajo costo de la mano de obra, la buena calidad en infraestructura y lo más importante, las políticas estatales. El estímulo fiscal y las facilidades para instalar empresas, que eran dispares entre región y región, estimularon a las empresas para destinar sus inversiones (Zebregs \& Tseng, 2002).

A partir de 1992 las reformas en su tercera etapa profundizarán el proceso de apertura y de transición económica. Concentrados en cinco puntos estas políticas tendieron a incrementar el monto y la importancia de las IED en el entramado productivo local:

1) El gobierno relajó las políticas restrictivas para permitir la participación de firmas extranjeras en la industria de servicios. Esto desató un optimismo para invertir y muchas firmas dispusieron sus activos en sectores como retail, consultorías y servicios.

2) Luego de la adopción del experimento de arrendamiento de tierras, hubo una oleada de inversiones inmobiliarias por corporaciones extranjeras.

3) Se firmaron proyectos para la inversión en infraestructura como autopistas, plantas de energía, puertos y aeropuertos. Debido a la escasez de fondos locales, el gobierno solicitaba préstamos en el extranjero para este tipo de obras. La modernización de la infraestructura resultó una de las mayores prioridades del gobierno.

4) Hubo una de privatización de las empresas estatales, destinado a convertir las empresas en joint ventures de capitales mixtos, mediante el establecimiento de nuevas participaciones o por arrendamiento a inversores extranjeros.

5) Desde 1991 el Consejo de Estado aprobó el establecimiento de doce zonas francas con la intención de promover el comercio y la inversión (Mee-kau, 1993).

Las políticas destinadas a la atracción de las IED tuvieron como resultado la llegada de inversiones millonarias que se materializaron en 324.000 proyectos. De esta manera el principal modo de inversión 
extranjera se realizó mediante los JV. Los JV permitieron cierto control sobre las inversiones y una oportunidad para que las empresas locales hicieran un uso estratégico de la IED. Acorde a los proyectos de asociaciones existieron tres principales vías para las IED. El primero fue el Equity Joint Venture (EJV) que se trataba de la creación de una empresa a través de inversiones conjuntas entre capitales locales e internacionales con limitadas obligaciones en las ganancias y en las perdidas. El segundo el Contractual Joint Venture (CJV), conocido como producción cooperativa, en el cual el socio extranjero proveía la tecnología y los materiales estratégicos mientras que la parte china otorgaba facilidades para la instalación, los materiales, servicios, logística y mano de obra. El tercero llamado Wholly Owned Subsidiary (OWS), o subsidiario de propiedad absoluta, en el cual el extranjero era el dueño y responsable de la totalidad de la empresa (Yuan \& Tsai, 2000).

Gracias a estas reformas, desde 1990 a 1995, las empresas internacionales instaladas en el país tuvieran un crecimiento del 40 por ciento anual en la participación del comercio exterior chino, mientras que las empresas locales tuvieron un crecimiento anual del 21 por ciento. Para 1995 las firmas internacionales fueron responsables del 31 por ciento de las exportaciones chinas; mientras que en 1998 este porcentaje se había incrementado al 48,67 por ciento (Yuan \& Tsai, 2000).

El resultado de las políticas estatales implementadas por el gobierno chino, desde 1992, tuvieron éxito en el desarrollo industrial del país. Las IED se focalizaron en las inversiones productivas, nuevos proyectos (greenfield) y las asociaciones llamadas joint ventures. La asociación lograda en China con el capital extranjero fue lo que le permitió incrementar la capacidad productiva. Si bien las empresas extranjeras tuvieron un peso creciente en la economía durante los noventa, no se tradujo en la extranjerización de la economía china, sino que gracias a la planificación de políticas estatales se logró consolidar un proceso de desarrollo basado en la producción y la exportación de manufacturas.

\section{Conclusión}

Tras el fin de la Guerra Fría se afianzó como idea indiscutida que el desarrollo y el crecimiento económico solo era posible gracias a las instituciones liberales y a las reformas económicas neoliberales promercado que facilitaran la libre circulación del capital. Los países latinoamericanos, en especial la Argentina, tras la crisis del Estado durante los ochenta y bajo los postulados del Consenso de Washington, implementaran una serie de reformas neoliberales tendientes a reducir y "modernizar el Estado" para atraer las IED.

La reforma del Estado que efectuará Argentina a partir de 1991 y la consecuente liberalización económica y atracción de capitales, tendrá como resultado la transnacionalización y una mayor vulnerabilidad de la economía hacia los vaivenes de la situación económica internacional. Las IED solo fueron posibles gracias a las facilidades otorgadas y a las privatizaciones de las empresas estatales.

China y su experiencia con las IED durante los noventas rompen con el esquema neoliberal. Las reformas encaradas a partir de 1992, enmarcadas dentro de un contexto internacional favorable al neoliberalismo, no redujeron la participación del Estado, sino que afianzaron la calidad en la intervención. Las estrategias estatales para la atracción de IED tuvieron como fin el incremento de las capacidades productivas del país, mediante infraestructura, transferencia de tecnología e inversiones productivas. 
Estrategias estatales para las Inversiones Extranjeras Directas ¿Qué puede aprender Argentina de las reformas económicas en China?

Si bien las ventajas ofrecidas por China no pueden ser comparables con las de Argentina, si podemos establecer un punto de comparación en cuanto al rol del Estado para el diseño de estrategias para las IED. En Argentina el contexto de auge del neoliberalismo el achicamiento y retirada del Estado se entendió como la clave para permitir la llegada de las IED y alcanzar el desarrollo. Por su parte en China el concepto de reforma económica fue entendido como el desarrollo de las capacidades estatales para amoldarse y transformarse en relación a las reformas económicas. La planificación y las medidas de incentivo fueron fundamentales para el desarrollo industrial de China, políticas que lejos de disminuir el rol del Estado, lo consolidaron como un actor clave.

Ambos casos confluirán en el punto en que fueron Estados que requerían un proceso de reformas estatales acorde a las demandas económicas del momento, afín con la idea de neoliberalismo económico promovido durante esos años. El cambio en cuanto al funcionamiento del Estado y los objetivos planteados significaron en ambos casos, cierta ruptura con el pasado. De esta manera el gobierno chino profundizó su proceso de apertura económica, en tanto que el Estado argentino culminó con un periodo de intervención económica con las privatizaciones de empresas públicas.

En esta confluencia las IED jugaron un rol clave que en ambos países se entendieron como indispensables. Si lo analizamos las inversiones representaron un cambio paradigmático en la transformación económica que gracias a las reformas se dieron en esos años. No se puede analizar el desempeño tanto de la economía China como de Argentina sino es a través de la llegada de una importante suma de inversiones que sin duda representaron e indujeron un cambio en sus estructuras económicas.

Pero es en los resultados en donde se pueden encontrar las divergencias entre los procesos. Y es que estos resultados precisamente fueron posible por las políticas de reforma estatal que ambos países llevaron a cabo. Como hemos visto en este artículo, la consolidación de las funciones del Estado a través de una burocracia estatal más eficiente es clave para el desarrollo de políticas que a largo plazo y que buscan orientar a las IED acorde a los intereses locales. En este sentido, como se ha podido analizar, las IED en un marco de promoción y dirección, como lo es el caso chino, pueden resultar positivas. De lo contrario, las reformas a través del debilitamiento del aparato estatal y la ausencia de políticas hacia las IED, como el caso argentino pueden conducir al fracaso. La sola privatización de las empresas públicas y la entrada de capitales a través de este sistema fue uno los factores responsables del quiebre del Estado Argentino en el 2001.

Si bien los puntos de partida, así como las estructuras estatales y económicas previas no eran similares, los procesos si pueden considerarse similares debido al contexto urgente de reforma para adaptarse la nueva situación internacional (de globalización económica) y ante el advenimiento de inversiones extranjeras. En este aspecto, el éxito del estado chino con el fortalecimiento del aparato estatal y el consecuente éxito económico gracias a las políticas estatales para las IED es que resulta, salvo los matices, un caso para analizar y comparar.

El desarrollo económico se presenta en el caso chino como un caso atípico. Ha demostrado que no necesariamente instituciones de corte liberal pueden conducir al desarrollo económico obteniendo ventajas del contexto internacional a través de las IED. Si bien puede resultar difícil el caso para imitar, 
Latin American Journal of Trade Policy 3 (2019) - Universidad de Chile

no podemos omitir la importancia que ha tenido el Estado como actor fundamental. Y es allí donde el caso paradigmático se vuelve importante como proceso de aprendizaje, ya que definitivamente toda reforma estatal debe estar orientada a fortalecer las funciones del estado y con ello las políticas económicas a largo plazo y de esta manera utilizar a las IED para apuntalar el desarrollo económico.

\section{Referencias}

Basualdo, E. (2006). La reestructuración de la economía argentina durante las últimas décadas de la sustitución de importaciones a la valorización financiera. In E. Basualdo \& E. Arceo (Eds.), Neoliberalismo y sectores dominantes. Tendencias globales y experiencias nacionales (pp. 123-177). Buenos Aires: CLACSO.

Benecke, D., \& Nascimento, R. (2003). El consenso de Washington revisado. Diálogo Político, 2003(2), 1330.

Brooks, S., \& Wohlforth, W. (2001). Power, globalization, and the end of the Cold War: Reevaluating a landmark case for ideas. International Security, 25(3), 5-53.

Cruz Soto, L. A. (2002). Neoliberalismo y globalización económica. Algunos elementos de análisis para precisar los conceptos. Contaduría y Administración(205).

Escudé, C. (2006). Festival de licuaciones. Causas y consecuencias de la pobreza en Argentina. Buenos Aires: Lumiere.

Fukuyama, F. (1992). The End of History and the Last Man. New York: Macmillan.

Granato, L., \& Oddone, C. N. (2007). La protección internacional del inversor extranjero a través de los acuerdos bilaterales de inversión. Tendencias, 8(2), 43-66.

Harvey, D. (2007). A brief history of neoliberalism. USA: Oxford University Press.

Huyghebaert, N., \& Quan, Q. (2011). Ownership dynamics after partial privatization: Evidence from China. The Journal of Law Economics Letters, 54(2), 389-429.

Jung, J.-Y. (2008). Retreat of the state? Restructuring the Chinese central bureaucracies in the era of economic globalization. China Review, 105-125.

Kulfas, M., Porta, F., \& Ramos, A. (2002). Inversión extranjera y empresas transnacionales en la economía argentina (Vol. LC/BUE/L.179 - LC/L.1766-P): CEPAL.

Kulfas, M., Portas, F., \& Ramos, J. (2002). La inversión extranjera en Argentina a fines del siglo XX. Paper presented at the Seminario Regional CEPAL/UNCTAD sobre Políticas de Inversión Extranjera Directa en América Latina Santiago de Chile.

Long, G. J. (2005). China's policies on FDI: Review and evaluation.

Mee-kau, N. (1993). FDI in China, trends, investments, performance, policies and prospcets. In J. Yushek \& M. Brosseau (Eds.), China Review. Hong Kong: Chinese University Press.

Porta, F., \& Ramos, A. (2002). Inversión extranjera directa y reformas estructurales en la Argentina. Aportes para la Integración Latinoamericana, 8.

Venugopal, R. (2015). Neoliberalism as concept. Economy Society, 44(2), 165-187.

Wang, X. (1998). Whither Troubled Chinese State-owned Enterprises. In J. Cheng (Ed.), China Review 1998 (pp. 363-394). Hong Kong: Chinese University Press.

Yuan, L., \& Tsai, T. (2000). Foreign Direct Investment in China. In China Review. Hong Kong: Chinese University Press.

Zebregs, H., \& Tseng, W. (2002). Foreign direct investment in China: some lessons for other countries: International Monetary Fund. 